\title{
Editorial: New Generation of Psychotherapies Inspired by Cognitive Neuroscience Development: Emergence of Neuro- crossnark cognitive Therapies
}

Citation: Naji, B., \& Ekhtiari, H. (2016). New generation of psychotherapies inspired by cognitive neuroscience development: Emergence of neurocognitive Therapies. Basic and Clinical Neuroscience, 7(3), 179-184. http://dx.doi.org/10.15412/J.BCN.03070301

: http://dx.doi.org/10.15412/J.BCN.03070301

Psychological interventions, which have been designed to change human interactions with themselves and the environment, have a long history. Currently, more than 500 schools exist in the field of psychotherapeutic interventions (Roth \& Fonagy, 2005). Design, innovation, introduction, and intervention method of each school are based on some theoretical frameworks and experimental evidence. This empirical evidence might be derived from the personal experiences of one therapist or a group of therapists, through scientific experimental trial(s), or organized networks of evidence-based empirical knowledge (Walker, 1957). Perhaps the "behavioral interventions" based on the rules of classical and operant conditioning are the best example for this type of "evidence-based" interventions with a background of well-established empirical studies.

Owning to the complexity of the human brain and its cognitive processes as well as the influences of environmental factors such as family, language, and culture, the community of psychotherapists have neglected "behavioral interventions" as the dominant stream despite their well-structured scientific methodologies (Donohue \& Fryling, 2007). However, interventions based on the individual experiences of elite therapists (such as analytical psychological interventions) have gained more attention in the community (Fonagy, 2003).

The emergence of cognitive-behavioral intervention and its emphasize on values, perceptions, and thoughts was an innovation in the field of psychotherapy to address the complexities of human cognition. It was also a response to inductive reasoning of conventional behavioral therapy (Wilson \& Clarck, 1999).

Behavioral interventions might be considered as the first wave of evidence-based psychotherapy, versus cognitivebehavioral interventions which was the second wave.

Incomplete definition of "cognition" in the second wave created the ground for emerging a new branch of psychotherapy that further studied and refined different concepts such as insight or language (Kovescses \& Radden, 2009; Hatfield, 2009; MacKay, 1987). Practically, acceptance and commitment therapy (ACT) is the most popular school among followers of this new movement (Zettle, 2005). The authors of this article do not consider a third wave in the context of this group of psychotherapies and believe that this new group can be regarded as the continuation of the second wave.

Over recent years, development of the cognitive sciences and utilization of new technologies such as brain mapping and stimulation, has empowered psychologists to acquire vast amount of knowledge about the brain and its functions. Cognition is no more considered a separate determinant of human behavior in accompany with personality, affection, language, and values. Now, cogrtition includes all data being processed and produced in the human brain. It also comprises the development of data outside the human brain and its processing in the human body and environment (Stufflebeam \& Rosen, 2007).

Although the methods of brain mapping are still in their initial stages of development, many believe that the revolutionary role of brain mapping on decoding the science of psychology likens the effect of using microscope in biology or telescope in astronomy (Myers \& De Wall, 2015).

The authors of this editorial believe that further research in cognitive neuroscience with emphasis on its multidisciplinary nature with anthropology and linguistics can set the basis for a new generation of scientific-based psychotherapies that is neither narrow-banded like behavioral therapy nor affected by the weak points of cognitive-behavioral therapies. Perhaps this third generation can be called "neurocognitive psychotherapy".

The ability to enter the "black box" of human mind and introduction of "science of change", will provide a new generation of psychotherapies with the ten following features: 


\section{Considering Quantitative Approach to Un- conscious Cognitive Processes}

Cognitive neuroscience has provided us with evidence indicative of our insufficient knowledge about the brain and its processes. Now, we know that a lot of brain functions have not yet been discovered; in the meantime, the effect of many unconscious processes on human behavior is undeniable. However, it may be argued that what psychoanalysts tried to describe, just with their genius minds, can now be examined by the novel methods of cognitive neuroscience. This is evident from the formation and development of neuropsychoanalysis (Cusumano \& Raz, 2014; Vaslamatzis, 2007). In this context, targeted cognitive interventions on unconscious processes such as memory, habitual behaviors, intuitive decisions, long-term memory, arousal processing, etc. can lead to the treatment of diseases such as depression, anxiety, obsessive disorders, or addiction (Whitehead, 2005).

\section{Converging with Experimental Sciences, Dealing with Biology and Mathematics}

Experimental sciences cannot be confined within the borders of conventionally defined disciplines such as chemistry, physics, or biology. Mathematics as the logical framework of all experimental sciences serves as the communication language amongst scientists active in these fields. Neuroscience-based psychotherapy provides exclusive opportunities to make dialogues with different levels of human biological infrastructures such as proteins, genetics, epigenetics, organelles, cells, and organs. Besides, mathematics can monitor and quantify interventions utilizing computational neurocognitive models (Gabbiani \& Cox, 2010).

\section{Considering the Effects of Environmental Factors in the Context of Cognitive Science}

In cognitive science, the human environment such as culture, religion, conceptual values, and family-social interactions are considered effective factors on the brain's cognitive processes. Even some scientists in this field focus on studying extracerebral cognitive processing like embodiment, and the interactions between the humans and their environment. Introduction of these socio-spiritual factors in the design of neurocognitive models provides a holistic approach in evaluations and interventions (Lambert, Nelson, Jovanovic, \& Cerdá, 2015; Esch \& Stefano, 2010; Fingelkurts \& Fingelkurts, 2009).

\section{Emerging New Interventions Based on Grow- ing Knowledge of Cognitive Neuroscience}

Cognitive neuroscience has revealed the infinite extent of underlying neurological mechanisms of mental health disorders, especially through brain mapping, cognitive assessment, and modeling. Brain mapping was initially utilized as "modern phrenology" to study cerebral structures and functions (Sohrabi \& Brook, 2005). However, in interaction with cognitive interventions, it is now capable of proposing novel ideas for altering structures and functions of different regions and networks in the brain, resulting in the improvement and management of the mental health disorders (Dichter, Sikich, Song, Voyvodic, \& Bodfish, 2012). For example, after discovery of some disorders in the interoceptive processes located in the anterior cingulate cortex and anterior insula via neuroimaging studies among some overweight people, some innovative ideas were introduced for designing cognitive interventions aiming to improve the interoception for weight reduction (Frank, Kullmann, \& Veit, 2013). Preliminary use of these interventions with methods such as interoceptive exercises and augmented or supported meditation has resulted in appraisable outcomes.

\section{Predicting the Effectiveness and Outcomes of Interventions and Their Individualization with Quantitative Measures Derived from Cognitive Neuroscience}

Choosing an effective intervention is based on patient's individual characteristics. Also, monitoring long-term efficacy of the intervention is considered a serious challenge in the field of psychotherapy. Recently published literature, presents the significant capability of brain mapping techniques in the prediction of the efficacy of various treatments in different groups of patients with mental health disorders by studying the activity of different brain areas and circuits (Galderisi, 2002; lbrich \& Arns, 2013). Inspired by the rapid development of brain mapping, Neuroimage, one of the most prestigious journals in the field of brain imaging, exclusively dedicated one of its issues in 2016 to the evaluation of effectiveness of the psychotherapies by brain mapping under the title of "Neuroimaging Mechanisms of Behavior Change".

\section{Combining Targeted Psychotherapy In- terventions with Medications and Electrical Stimulations}

In regard to mental health disorders, cognitive neuroscience is the cross-road between psychotherapy, electrical stimulation, and pharmacotherapy. For instance, brain imag- 
ing indicates that hypoactivity of the right lateral prefrontal cortex predicts the mood state in patients with depression (Rive et al., 2013). In addition, cognitive intervention suggests that attention training with acoustic stimuli (known as paced auditory serial addition training) could increase the activity of this area of the brain (Audoin et al., 2005). Therefore, $2 \mathrm{~mA}$ transcranial direct current stimulation (tDCS) for 20 minutes can increase this activity (Weber et al., 2014). Moreover, studies have shown that the serotonergic group of antidepressants (i.e. selective serotonin reuptake inhibitors) prolongs the duration and effectiveness of electrical brain stimulation. This knowledge can bring a combinative therapeutic plan using cognitive intervention, medication, and electrical stimulation for the treatment of depressed patients. Most recently, these combined interventions received serious attention in ongoing clinical trials (Pilling \& Roiser, 2015).

\section{Implementing Cognitive Enhancement} Techniques to Induce Cognitive Changes (Integration of Psychotherapy and Rehabilitation)

Rehabilitative interventions targeting cognitive deficits caused by brain injuries such as trauma, vascular disorders (e.g. stroke) (Tsaousides \& Gordon, 2009), and neurological inflammations (e.g. multiple sclerosis) (Chiaravalloti, Genova, \& DeLuca, 2015) could be considered important areas in the field of cognitive interventions (Cicerone et al., 2002). Cognitive rehabilitation has provided exciting ideas for improving the capabilities of the brain even in healthy subjects (Enriquez-Geppert, Huster, \& Herrmann, 2013; Gruzelier, 2014).

But the main objective of psychotherapy is to "modify" cognitive processing to change emotions or behaviors in clients. Development of cognitive neuroscience has practically combined these two areas of modification and enhancement. Hereby, it is possible to combine cognitive enhancement or rehabilitative interventions with psychotherapy. Organizing cognitive interventions with focus on the enhancement of attention and cognitive inhibition, strengthening interoception, promotion of insight and metacognition, and trying to improve the outcomes of psychotherapy are accomplishments of this promising combined therapy (Hillis et al., 2015).

\section{Reclassifying Mental Health Disorders in the Framework of Neurocognitive Endophenotypes}

Accurate description and classification of signs and symptoms (i.e. external phenotype or exophenotype) has been the conventional method in psychiatric semiotics in the $20^{\text {th }}$ century. But a more thorough understanding of biological neurocognitive infrastructures of psychiatric disorders such as addiction, depression, schizophrenia, or even obesity using cognitive technologies, has provided an opportunity to reorganize the classification of these disorders (Ziauddeen, Alonso-Alonso, Hill, Kelley, \& Khan, 2015; Saunders, Richard, \& Janak, 2015). Revealing hidden cognitive disorders or cognition based endophenotypes such as impulse regulatory disorders, decision making deificit, judgment dysfunction, cognitive bias, or stress dysregulation can lead to a better understanding of psychiatric disorders and implementation of effective interventions (Sahakian, et al., 2015; Faingold \& Blumenfeld, 2015).

\section{Creating a Matrix of Environmental, Neu- ral, and Cognitive Interventions Emphasizing on Gaps for Research Needs}

One of the most important results of the cognitive neuroscience adoption as the foundation of psychotherapies is to realize our vast lack of knowledge in the mechanisms of effective psychological interventions. The introduction of the project "brain research through advancing innovative neurotechnologies" (BRAIN), launched by the President of the United States, Mr. Obama, with a fund of over \$ 2 billion as well as human brain projects (HBP) in Europe with more than $\underline{€} 2$ billion funding, are examples of this effort to create and organize a research matrix at the level of national policies (White House Office of the Press Secretary, 2013; The HBP Board of Directors, 2014).

\section{Approaching "Brain Health" with a Holis- tic View}

Differentiation of brain diseases into psychiatric and neurologic disorders, which is due to the complexity in this field of medicine, challenges the accurate understanding and treatment of these diseases. In this regard, a holistic approach to "brain health" and prevention of cerebral injuries and malfunctions in the light of neuroscience can accelerate the fusion process of these two medical specialties. This approach will certainly result in the improvement of brain health and quality of people's life, with more emphasis on the prevention of diseases, provision of effective treatment, and continuity of brain rehabilitation. Meanwhile, we should accept that overestimation of the reliability of our current premature knowledge of neuroscience, may ultimately result in our failure in the management of brain health disorders during real clinical practice.

Regarding the potentiality of psychotherapeutic interventions with cognitive neuroscience models in contribution to 
the mental health of societies in the future, it is our duty as experts in this field to initiate and promote an integrated global campaign to encourage politicians, legislators, and economists to consider funding policies for supporting this promising and progressing movement. Cognitive science could provide a common platform for technologies, methods, and terminologies of different disciplines (from psychology to neuroscience) to contribute in this movement. Reaching a worldwide consensus upon the importance of research in this field not only can promote the mental health of the societies, but also aid government financially in the long term. Hopefully, the collaboration of neuroscientists and psychologists with policy makers to prioritize budget planning supporting translational cognitive neuroscience studies to inspire psychotherapy will result in attaining unprecedented horizons in the management and treatment of mental health disorders and improving the quality of life for millions.

\section{Borzooyeh Naji $1^{1}$, Hamed Ekhtiari ${ }^{1,2,3^{*}}$}

1.Translational Neuroscience Program, Institute for Cognitive Science Studies, Tehran, Iran

2. Neurocognitive Laboratory, Iranian National Center for Addiction Studies, Tehran University of Medical Sciences, Tehran, Iran.

3. Neuroimaging and Analysis Group (NIAG), Research Center for Molecular and Cellular Imaging (RCMCI), Tehran University of Medical Sciences, Tehran, Iran.

\section{* Corresponding Author:}

Hamed Ekhtiari, MD

Address: Translational Neuroscience Program, Institute for Cognitive Science Studies, Tehran, Iran.

E-mail:h_ekhtiari@razi.tums.ac.ir

\section{References}

Audoin, B., Ibarrola, D., Au Duong, M. V., Pelletier, J., ConfortGouny, S., Malikova, I., et al. (2005). Functional MRI study of PASAT in normal subjects. Magnetic Resonance Materials in Physics, Biology and Medicine (MAGMA), 18(2), 96-102.

Chiaravalloti, N. D., Genova, H. M., \& DeLuca, J. (2015). Cognitive Rehabilitation in Multiple Sclerosis: The Role of Plasticity. Frontiers in Neurology, 6, 67.

Cicerone, K. D., Dahlberg, C., Malec, J. F., Langenbahn, D. M., Felicetti, T., Kneipp, S., et al. (2002). Evidence-based cognitive rehabilitation: updated review of the literature from 1998 through 2002. Archives of Physical Medicine and Rehabilitation, 86(8):1681-92.

Cusumano, E. P., \& Raz, A. (2014). Harnessing psychoanalytical methods for a phenomenological neuroscience. Frontiers in Psychology, 5, 334
Dichter, G. S., Sikich, L., Song, A., Voyvodic, J., \& Bodfish, J. W (2012). Functional neuroimaging of treatment effects in psychiatry: Methodological challenges and recommendations. International Journal of Neuroscience, 122(9), 483-93.

Donohue, W., \& Fryling, M. (2007). How Has Applied Behavior Analysis and Behavior Therapy Changed? An Historical Analysis of Journals. Behavior Analyst Today, 8(1), 52.

Enriquez-Geppert, S., Huster, R. J., \& Herrmann, C. S. (2013). Boosting brain functions: Improving executive functions with behavioral training, neurostimulation, and neurofeedback. International Journal of Psychophysiology, 88(1), 1-16.

Esch, T., \& Stefano, G. B. (2010). The neurobiology of stress management. Neuroendocrinology letters, 31(1), 19-39.

Faingold, C. L., \& Blumenfeld, H. (2015). Targeting Neuronal Networks with Combined Drug and Stimulation Paradigms Guided by Neuroimaging to Treat Brain Disorders. Neuroscientist, 21(5), 460-474.

Fingelkurts, A. A., \& Fingelkurts, A. A. (2009). Is our brain hardwired to produce God, or is our brain hardwired to perceive God? A systematic review on the role of the brain in mediating religious experience. Cognitive Processing, 10(4), 293-326.

Fonagy, P. (2003). Psychoanalysis today. World Psychiatry, 2(2), 73-80.

Frank, S., Kullmann, S., \& Veit, R. (2013). Food related processes in the insular cortex. Frontiers in Human Neuroscience, 7, 499.

Gruzelier, J. H. (2014). EEG-neurofeedback for optimizing performance. III: a review of methodological and theoreticalconsiderations. Neuroscience \& Biobehavioral Reviews, 44, 159-82.

Hatfield, G. C. (2009). Perception and cognition: Essays in the philosophy of psychology. New York: Oxford University Press.

Hillis, J. D., Leonhardt, B. L., Vohs, J. L., Buck, K. D., Salvatore, G., Popolo, R., et al. (2015). Metacognitive reflective and insight therapy for people in early phase of a schizophrenia spectrum disorder. Journal of Clinical Psychology, 71(2), 125-35.

lbrich, S., \& Arns, M. (2013). EEG biomarkers in major depressive disorder: discriminative power and prediction of treatmentresponse. International Review of Psychiatry, 25(5), 604-18.

Gabbiani, F., \& Cox, S. J. (2010). Mathematics for Neuroscientists. London, Elsevier: Academic Press.

Galderisi, S. (2002). Clinical applications of pharmaco-EEG in psychiatry: the prediction of response to treatment with antipsychotics. Methods \& Findings in Experimental \& Clinical Pharmacology, 24, 85-9.

Kovescses, Z., \& Radden, G. (2009). Metonymy: Developing a cognitive linguistic view. Cognitive Linguistics, 9(1), 37-78.

Lambert, K. G., Nelson, R. J., Jovanovic, T., \& Cerdá, M. (2015). Brains in the city: Neurobiological effects of urbanization. Neuroscience \& Biobehavioral Reviews, 58, 107-122.

MacKay, D. G. (1987). The organization of perception and action: A theory for language and other cognitive skills. New York: SpringerVerlag.

Myers, D. G., \& De Wall, C. N. (2015). Psychology (11 ${ }^{\text {th }}$ ed.). New York: Worth Publishers. 
Pilling, S., \& Roiser, J. P. (2015). Non-invasive Brain Stimulation and Cognitive Processing in Depression, this study is currently recruiting participants. London: University College.

Rive, M. M., van Rooijen, G., Veltman, D. J., Phillips, M. L., Schene, A. H., \& Ruhé, H. G. (2013). Neural correlates of dysfunctional emotion regulation in major depressive disorder: A systematic review of neuroimaging studies. Neuroscience \& Biobehavioral Reviews, 37(10), 2529-53.

Roth, A., \& Fonagy, P. (2005). What Works for Whom? A Critical Review of Psychotherapy Research ( $2^{\text {nd }}$ ed.). New York: Guilford Press.

Sahakian, B. J., Bruhl, A. B., Cook, J., Killikelly, C., Savulich, G., Piercy, T., et al. (2015). The impact of neuroscience on society: Cognitive enhancement in neuropsychiatric disorders and in healthy people. Philosophical transactions of the Royal Society of London. Series B, Biological Sciences, 370(1677), 20140214.

Saunders, B. T., Richard, J. M., \& Janak, P. H. (2015). Contemporary approaches to neural circuit manipulation and mapping: Focus on reward and addiction. Philosophical transactions of the Royal Society of London. Series B, Biological Sciences, 370(1677), 20140210.

Sohrabi, A., \& Brook, A. (2005). Functional neuroimaging and its implications for cognitive science: Beyond phrenology and localization. In Proceedings of the Twenty-Seventh Annual Meeting of the Cognitive Science Society (pp. 2044-2049). Italy: Stresa.

Stufflebeam, S. M., \& Rosen, B. (2007). Mapping Cognitive Function. Neuroimaging Clinics of North America, 17(4), 469-484

The HBP Board of Directors and the HBP Executive Committee, (2014). The Vital Role of Neuroscience in the Human Brain Project. The Human Brain Project. Retrieved from https://www. humanbrainproject.eu/documents/10180/17646/HBP-Statement.090614.pdf.

Tsaousides, T., \& Gordon, W. A. (2009). Cognitive rehabilitation following traumatic brain injury: assessment to treatment. Mount Sinai Journal of Medicine: A Journal of Translational and Personalized Medicine, 76(2), 173-81.

Vaslamatzis, G. (2007). Framework for a new dialogue between psychoanalysis and neurosciences: Is the combined neuro-psychoanalytic approach the missing link? Philosophy, Ethics, and Humanities in Medicine, 2(1), 25.

Walker, N. (1957). A Short History of Psychotherapy in Theory and Practice. Abingdon: Routledge and Kegan Paul.

Weber, M. J., Messing, S. B., Rao, H., Detre, J. A., \& ThompsonSchill, S. L. (2014). Prefrontal transcranial direct current stimulation alters activation and connectivity in cortical and subcortical reward systems: A tDCS-fMRI study. Human Brain Mapping, 35(8), 3673-86.

Whitehead, C. C. (2005). Toward a "new" paradigm of therapeutic action: neuro-psychoanalysis and downward causation. Journal of the American Academy of Psychoanalysis and Dynamic Psychiatry, $33(4), 637-56$.

Wilson, G. T., \& Clark, D. M. (1999). Cognitive behaviour therapy: Evolution and prospects-A festschrift in honour of Dr. S. Rachman. Behaviour Research and Therapy, 37(1), S1-191.

Ziauddeen, H., Alonso-Alonso, M., Hill, J. O., Kelley, M., \& Khan, N. A. (2015). Obesity and the Neurocognitive Basis of Food Reward and the Control of Intake. Advances in Nutrition, 6(4), 474-86.
Zettle, R. D. (2005). The Evolution of a Contextual Approach to Therapy: From Comprehensive Distancing to ACT. International Journal of Behavioral Consultation and Therapy, 1(2), 77 . 
